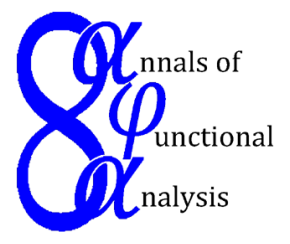

Ann. Funct. Anal. 7 (2016), no. 1, 9-16

http://dx.doi.org/10.1215/20088752-3158134

ISSN: 2008-8752 (electronic)

http://projecteuclid.org/afa

\title{
HOMOGENEOUS SPACES AND SQUARE-INTEGRABLE REPRESENTATIONS
}

\author{
FATEMEH ESMAEELZADEH ${ }^{1^{*}}$ and RAJAB ALI KAMYABI GOL ${ }^{2}$ \\ Dedicated to Professor Anthony To-Ming Lau \\ Communicated by O. Christensen
}

\begin{abstract}
For a locally compact group $G$ and a compact subgroup $H$ of $G$, the square-integrable representations of group $G$ and homogeneous space $G / H$ are described. Also, the connection between the existence of admissible wavelets for locally compact groups and their homogeneous spaces is compared. Moreover, some properties of admissible wavelets and wavelet constants for homogeneous space $G / H$ are investigated, when $G$ is unimodular.
\end{abstract}

\section{INTRODUCTION AND PRELIMINARIES}

For a locally compact group $G$, it is well known that a continuous unitary representation $\pi$ of $G$ is called square integrable if there exists a nonzero vector $\zeta$ in Hilbert space $\mathcal{H}$ such that

$$
\int_{G}|\langle\pi(x) \zeta, \zeta\rangle|^{2} d \lambda(x)<\infty
$$

in which $\lambda$ is a left Haar measure. Such a unital vector $\zeta$ is called an admissible vector. The wavelet constant associated to the admissible wavelet is denoted by $c_{\zeta}$ and defined by

$$
c_{\zeta}=\int_{G}|\langle\zeta, \pi(x) \zeta\rangle|^{2} d \lambda(x)
$$

Copyright 2016 by the Tusi Mathematical Research Group.

Received Sep. 30, 2014; Accepted Dec. 22, 2014.

${ }^{*}$ Corresponding author.

2010 Mathematics Subject Classification. Primary 43A15; Secondary 43A85, 65T60.

Keywords. homogeneous space, square integrable, admissible wavelet, relatively invariant measure, strongly quasi-invariant measure. 


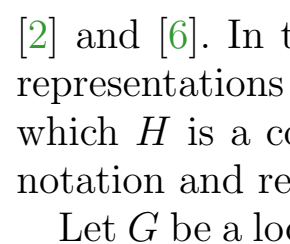

Ann. Funct. Anal. 7 (2016), no. 1, 9-16

http://dx.doi.org/10.1215/20088752-3158134

ISSN: 2008-8752 (electronic)

http://projecteuclid.org/afa

\title{
HOMOGENEOUS SPACES AND SQUARE-INTEGRABLE REPRESENTATIONS
}

\author{
FATEMEH ESMAEELZADEH ${ }^{1^{*}}$ and RAJAB ALI KAMYABI GOL ${ }^{2}$ \\ Dedicated to Professor Anthony To-Ming Lau \\ Communicated by O. Christensen
}

\begin{abstract}
For a locally compact group $G$ and a compact subgroup $H$ of $G$, the square-integrable representations of group $G$ and homogeneous space $G / H$ are described. Also, the connection between the existence of admissible wavelets for locally compact groups and their homogeneous spaces is compared. Moreover, some properties of admissible wavelets and wavelet constants for homogeneous space $G / H$ are investigated, when $G$ is unimodular.
\end{abstract}

\section{INTRODUCTION AND PRELIMINARIES}

For a locally compact group $G$, it is well known that a continuous unitary representation $\pi$ of $G$ is called square integrable if there exists a nonzero vector $\zeta$ in Hilbert space $\mathcal{H}$ such that

$$
\int_{G}|\langle\pi(x) \zeta, \zeta\rangle|^{2} d \lambda(x)<\infty
$$

in which $\lambda$ is a left Haar measure. Such a unital vector $\zeta$ is called an admissible vector. The wavelet constant associated to the admissible wavelet is denoted by $c_{\zeta}$ and defined by

$$
c_{\zeta}=\int_{G}|\langle\zeta, \pi(x) \zeta\rangle|^{2} d \lambda(x)
$$

Copyright 2016 by the Tusi Mathematical Research Group.

Received Sep. 30, 2014; Accepted Dec. 22, 2014.

${ }^{*}$ Corresponding author.

2010 Mathematics Subject Classification. Primary 43A15; Secondary 43A85, 65T60.

Keywords. homogeneous space, square integrable, admissible wavelet, relatively invariant measure, strongly quasi-invariant measure. 


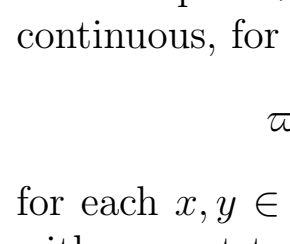

Ann. Funct. Anal. 7 (2016), no. 1, 9-16

http://dx.doi.org/10.1215/20088752-3158134

ISSN: 2008-8752 (electronic)

http://projecteuclid.org/afa

\title{
HOMOGENEOUS SPACES AND SQUARE-INTEGRABLE REPRESENTATIONS
}

\author{
FATEMEH ESMAEELZADEH ${ }^{1^{*}}$ and RAJAB ALI KAMYABI GOL ${ }^{2}$ \\ Dedicated to Professor Anthony To-Ming Lau \\ Communicated by O. Christensen
}

\begin{abstract}
For a locally compact group $G$ and a compact subgroup $H$ of $G$, the square-integrable representations of group $G$ and homogeneous space $G / H$ are described. Also, the connection between the existence of admissible wavelets for locally compact groups and their homogeneous spaces is compared. Moreover, some properties of admissible wavelets and wavelet constants for homogeneous space $G / H$ are investigated, when $G$ is unimodular.
\end{abstract}

\section{INTRODUCTION AND PRELIMINARIES}

For a locally compact group $G$, it is well known that a continuous unitary representation $\pi$ of $G$ is called square integrable if there exists a nonzero vector $\zeta$ in Hilbert space $\mathcal{H}$ such that

$$
\int_{G}|\langle\pi(x) \zeta, \zeta\rangle|^{2} d \lambda(x)<\infty
$$

in which $\lambda$ is a left Haar measure. Such a unital vector $\zeta$ is called an admissible vector. The wavelet constant associated to the admissible wavelet is denoted by $c_{\zeta}$ and defined by

$$
c_{\zeta}=\int_{G}|\langle\zeta, \pi(x) \zeta\rangle|^{2} d \lambda(x)
$$

Copyright 2016 by the Tusi Mathematical Research Group.

Received Sep. 30, 2014; Accepted Dec. 22, 2014.

${ }^{*}$ Corresponding author.

2010 Mathematics Subject Classification. Primary 43A15; Secondary 43A85, 65T60.

Keywords. homogeneous space, square integrable, admissible wavelet, relatively invariant measure, strongly quasi-invariant measure. 


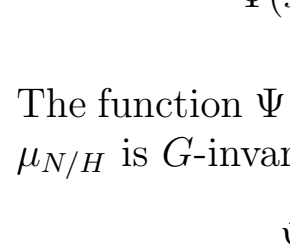

Ann. Funct. Anal. 7 (2016), no. 1, 9-16

http://dx.doi.org/10.1215/20088752-3158134

ISSN: 2008-8752 (electronic)

http://projecteuclid.org/afa

\title{
HOMOGENEOUS SPACES AND SQUARE-INTEGRABLE REPRESENTATIONS
}

\author{
FATEMEH ESMAEELZADEH ${ }^{1^{*}}$ and RAJAB ALI KAMYABI GOL ${ }^{2}$ \\ Dedicated to Professor Anthony To-Ming Lau \\ Communicated by O. Christensen
}

\begin{abstract}
For a locally compact group $G$ and a compact subgroup $H$ of $G$, the square-integrable representations of group $G$ and homogeneous space $G / H$ are described. Also, the connection between the existence of admissible wavelets for locally compact groups and their homogeneous spaces is compared. Moreover, some properties of admissible wavelets and wavelet constants for homogeneous space $G / H$ are investigated, when $G$ is unimodular.
\end{abstract}

\section{INTRODUCTION AND PRELIMINARIES}

For a locally compact group $G$, it is well known that a continuous unitary representation $\pi$ of $G$ is called square integrable if there exists a nonzero vector $\zeta$ in Hilbert space $\mathcal{H}$ such that

$$
\int_{G}|\langle\pi(x) \zeta, \zeta\rangle|^{2} d \lambda(x)<\infty
$$

in which $\lambda$ is a left Haar measure. Such a unital vector $\zeta$ is called an admissible vector. The wavelet constant associated to the admissible wavelet is denoted by $c_{\zeta}$ and defined by

$$
c_{\zeta}=\int_{G}|\langle\zeta, \pi(x) \zeta\rangle|^{2} d \lambda(x)
$$

Copyright 2016 by the Tusi Mathematical Research Group.

Received Sep. 30, 2014; Accepted Dec. 22, 2014.

${ }^{*}$ Corresponding author.

2010 Mathematics Subject Classification. Primary 43A15; Secondary 43A85, 65T60.

Keywords. homogeneous space, square integrable, admissible wavelet, relatively invariant measure, strongly quasi-invariant measure. 


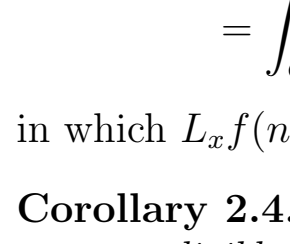

Ann. Funct. Anal. 7 (2016), no. 1, 9-16

http://dx.doi.org/10.1215/20088752-3158134

ISSN: 2008-8752 (electronic)

http://projecteuclid.org/afa

\title{
HOMOGENEOUS SPACES AND SQUARE-INTEGRABLE REPRESENTATIONS
}

\author{
FATEMEH ESMAEELZADEH ${ }^{1^{*}}$ and RAJAB ALI KAMYABI GOL ${ }^{2}$ \\ Dedicated to Professor Anthony To-Ming Lau \\ Communicated by O. Christensen
}

\begin{abstract}
For a locally compact group $G$ and a compact subgroup $H$ of $G$, the square-integrable representations of group $G$ and homogeneous space $G / H$ are described. Also, the connection between the existence of admissible wavelets for locally compact groups and their homogeneous spaces is compared. Moreover, some properties of admissible wavelets and wavelet constants for homogeneous space $G / H$ are investigated, when $G$ is unimodular.
\end{abstract}

\section{INTRODUCTION AND PRELIMINARIES}

For a locally compact group $G$, it is well known that a continuous unitary representation $\pi$ of $G$ is called square integrable if there exists a nonzero vector $\zeta$ in Hilbert space $\mathcal{H}$ such that

$$
\int_{G}|\langle\pi(x) \zeta, \zeta\rangle|^{2} d \lambda(x)<\infty
$$

in which $\lambda$ is a left Haar measure. Such a unital vector $\zeta$ is called an admissible vector. The wavelet constant associated to the admissible wavelet is denoted by $c_{\zeta}$ and defined by

$$
c_{\zeta}=\int_{G}|\langle\zeta, \pi(x) \zeta\rangle|^{2} d \lambda(x)
$$

Copyright 2016 by the Tusi Mathematical Research Group.

Received Sep. 30, 2014; Accepted Dec. 22, 2014.

${ }^{*}$ Corresponding author.

2010 Mathematics Subject Classification. Primary 43A15; Secondary 43A85, 65T60.

Keywords. homogeneous space, square integrable, admissible wavelet, relatively invariant measure, strongly quasi-invariant measure. 


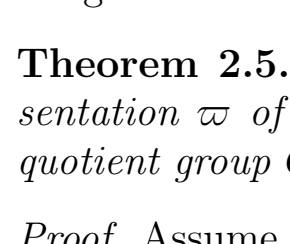

Ann. Funct. Anal. 7 (2016), no. 1, 9-16

http://dx.doi.org/10.1215/20088752-3158134

ISSN: 2008-8752 (electronic)

http://projecteuclid.org/afa

\title{
HOMOGENEOUS SPACES AND SQUARE-INTEGRABLE REPRESENTATIONS
}

\author{
FATEMEH ESMAEELZADEH ${ }^{1^{*}}$ and RAJAB ALI KAMYABI GOL ${ }^{2}$ \\ Dedicated to Professor Anthony To-Ming Lau \\ Communicated by O. Christensen
}

\begin{abstract}
For a locally compact group $G$ and a compact subgroup $H$ of $G$, the square-integrable representations of group $G$ and homogeneous space $G / H$ are described. Also, the connection between the existence of admissible wavelets for locally compact groups and their homogeneous spaces is compared. Moreover, some properties of admissible wavelets and wavelet constants for homogeneous space $G / H$ are investigated, when $G$ is unimodular.
\end{abstract}

\section{INTRODUCTION AND PRELIMINARIES}

For a locally compact group $G$, it is well known that a continuous unitary representation $\pi$ of $G$ is called square integrable if there exists a nonzero vector $\zeta$ in Hilbert space $\mathcal{H}$ such that

$$
\int_{G}|\langle\pi(x) \zeta, \zeta\rangle|^{2} d \lambda(x)<\infty
$$

in which $\lambda$ is a left Haar measure. Such a unital vector $\zeta$ is called an admissible vector. The wavelet constant associated to the admissible wavelet is denoted by $c_{\zeta}$ and defined by

$$
c_{\zeta}=\int_{G}|\langle\zeta, \pi(x) \zeta\rangle|^{2} d \lambda(x)
$$

Copyright 2016 by the Tusi Mathematical Research Group.

Received Sep. 30, 2014; Accepted Dec. 22, 2014.

${ }^{*}$ Corresponding author.

2010 Mathematics Subject Classification. Primary 43A15; Secondary 43A85, 65T60.

Keywords. homogeneous space, square integrable, admissible wavelet, relatively invariant measure, strongly quasi-invariant measure. 


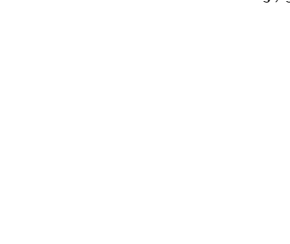

Ann. Funct. Anal. 7 (2016), no. 1, 9-16

http://dx.doi.org/10.1215/20088752-3158134

ISSN: 2008-8752 (electronic)

http://projecteuclid.org/afa

\title{
HOMOGENEOUS SPACES AND SQUARE-INTEGRABLE REPRESENTATIONS
}

\author{
FATEMEH ESMAEELZADEH ${ }^{1^{*}}$ and RAJAB ALI KAMYABI GOL ${ }^{2}$ \\ Dedicated to Professor Anthony To-Ming Lau \\ Communicated by O. Christensen
}

\begin{abstract}
For a locally compact group $G$ and a compact subgroup $H$ of $G$, the square-integrable representations of group $G$ and homogeneous space $G / H$ are described. Also, the connection between the existence of admissible wavelets for locally compact groups and their homogeneous spaces is compared. Moreover, some properties of admissible wavelets and wavelet constants for homogeneous space $G / H$ are investigated, when $G$ is unimodular.
\end{abstract}

\section{INTRODUCTION AND PRELIMINARIES}

For a locally compact group $G$, it is well known that a continuous unitary representation $\pi$ of $G$ is called square integrable if there exists a nonzero vector $\zeta$ in Hilbert space $\mathcal{H}$ such that

$$
\int_{G}|\langle\pi(x) \zeta, \zeta\rangle|^{2} d \lambda(x)<\infty
$$

in which $\lambda$ is a left Haar measure. Such a unital vector $\zeta$ is called an admissible vector. The wavelet constant associated to the admissible wavelet is denoted by $c_{\zeta}$ and defined by

$$
c_{\zeta}=\int_{G}|\langle\zeta, \pi(x) \zeta\rangle|^{2} d \lambda(x)
$$

Copyright 2016 by the Tusi Mathematical Research Group.

Received Sep. 30, 2014; Accepted Dec. 22, 2014.

${ }^{*}$ Corresponding author.

2010 Mathematics Subject Classification. Primary 43A15; Secondary 43A85, 65T60.

Keywords. homogeneous space, square integrable, admissible wavelet, relatively invariant measure, strongly quasi-invariant measure. 


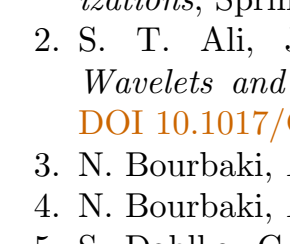

Ann. Funct. Anal. 7 (2016), no. 1, 9-16

http://dx.doi.org/10.1215/20088752-3158134

ISSN: 2008-8752 (electronic)

http://projecteuclid.org/afa

\title{
HOMOGENEOUS SPACES AND SQUARE-INTEGRABLE REPRESENTATIONS
}

\author{
FATEMEH ESMAEELZADEH ${ }^{1^{*}}$ and RAJAB ALI KAMYABI GOL ${ }^{2}$ \\ Dedicated to Professor Anthony To-Ming Lau \\ Communicated by O. Christensen
}

\begin{abstract}
For a locally compact group $G$ and a compact subgroup $H$ of $G$, the square-integrable representations of group $G$ and homogeneous space $G / H$ are described. Also, the connection between the existence of admissible wavelets for locally compact groups and their homogeneous spaces is compared. Moreover, some properties of admissible wavelets and wavelet constants for homogeneous space $G / H$ are investigated, when $G$ is unimodular.
\end{abstract}

\section{INTRODUCTION AND PRELIMINARIES}

For a locally compact group $G$, it is well known that a continuous unitary representation $\pi$ of $G$ is called square integrable if there exists a nonzero vector $\zeta$ in Hilbert space $\mathcal{H}$ such that

$$
\int_{G}|\langle\pi(x) \zeta, \zeta\rangle|^{2} d \lambda(x)<\infty
$$

in which $\lambda$ is a left Haar measure. Such a unital vector $\zeta$ is called an admissible vector. The wavelet constant associated to the admissible wavelet is denoted by $c_{\zeta}$ and defined by

$$
c_{\zeta}=\int_{G}|\langle\zeta, \pi(x) \zeta\rangle|^{2} d \lambda(x)
$$

Copyright 2016 by the Tusi Mathematical Research Group.

Received Sep. 30, 2014; Accepted Dec. 22, 2014.

${ }^{*}$ Corresponding author.

2010 Mathematics Subject Classification. Primary 43A15; Secondary 43A85, 65T60.

Keywords. homogeneous space, square integrable, admissible wavelet, relatively invariant measure, strongly quasi-invariant measure. 